\title{
NOTA SOBRE LA EVOLUCION \\ DE LOS SISTEMAS DE INFORMACION CONTABLE
}

JAVIER ZORNOZA BOY

\section{Introducción}

La aparición del libro de H. Thomas Johnson y Robert S. Kaplan La contabilidad de costes. Auge y caida de la contabilidad de gestión ${ }^{1}$, cuyo título puede resultar una sorpresa para lo que muchos estudiosos de la economía entienden qué es la contabilidad de gestión y por lo que sugiere de mal momento para aquella disciplina, nos lleva a plantearnos en el presente artículo las transformaciones experimentadas por el sistema de información que conocemos con el nombre de contabilidad y con el que, aunque no sea más que como usuarios, nos relacionamos cotidianamente.

Es sabido que, al menos durante el siglo xix y en lo que va transcurrido del siglo $\mathrm{xx}$, los sistemas de información con los que han operado las empresas de una cierta dimensión, y que les permitieron tanto el seguimiento de sus logros como el control de sus actividades, tomaron principalmente la forma de un sistema de información contable. Esto es, las empresas expresaron la mayor parte de los datos relativos a sus operaciones utilizando el lenguaje y ciertas convenciones desarrolladas desde algunas centurias antes por la contabilidad.

Sin embargo, puesto que al hablar de contabilidad empresarial estamos refiriéndonos a la elaboración de información sobre una entidad, interesa poner de relieve que dicha información resulta en parte impuesta por las propias características de la entidad y por su entorno económico, legal, tecnológico, etc. Históricamente se puede observar cómo nuevas demandas de información, debidas al surgimiento de nuevas actividades, han llevado a desarrollar los sistemas de información disponibles hasta ese momento.

De este modo, en el siglo XIX se han producido, como es bien sabido, los dos mayores estímulos al desarrollo de la disciplina contable tal y como la conocemos hoy día. El primero de ellos vino dado principalmente por el cre-

\footnotetext{
'Johnson y Kaplan (1988).
} 
cimiento de los mercados financieros, por el amplio desarrollo de las socie-

dades por acciones y por la regulación legal de estos procesos; lo que condujo en la lingläterra del siglo pasado al désarrollo de un conjunto de convenciones aptas para proporcionar a true and fair view del capital invertido en una empresa y de los resultados de sus operaciones ${ }^{2}$. Para que tal «imagen» contable de la compañía tuviera mayores garantías, se desarrolló también la revisión de cuentas independiente a la propia empresa. Como consecuencia de todo lo anterior, se convierten en moneda corriente los informes financieros auditados, dirigidos a usuarios externos a lo que es la propia dirección de la empresa (mercado de capitales, etc.). Dicha información continúa siendo extremadamente útil en nuestros días.

El segundo gran estímulo al desarrollo de la moderna contabilidad, que es, sin embargo, menos conocido que el anterior, tiene lugar al tiempo que se produce un proceso de concentración del capital en algunas industrias, con la integración horizontal de actividades que anteriormente se realizaban por diferentes empresas, extendiendo así el ciclo productivo que lleva a cabo la entidad; o bien al comenzar a operar empresas integradas verticalmente con procesos y productos dispares que estaban separados antes de la integración, y pasan a ser gestionados bajo la misma dirección. En todos estos casos, surge una necesidad interna de información sobre operaciones que antes de la integración se llevaban a cabo en el mercado (mercado de productos o mercado de capitales) y que después del proceso de integración van a pasar a ser dirigidos internamente, en la compañía, utilizando como «señales» los datos elaborados internamente para la gestión. Este proceso ha sido estudiado con cierta profundidad para los Estados Unidos ${ }^{3} \mathrm{y}$ abarca desde principios del siglo XIX con el surgimiento de los primeros molinos textiles, hasta el surgimiento de las empresas estructuradas en divisiones en la década de los años veinte de nuestro siglo. A lo largo de este período de tiempo los nuevos procesos de concentración industrial incentivaban el desarrollo de nuevos sistemas contables para la gestión interna, lo que es conocido en nuestros días como «Contabilidad de Costes», en un sentido amplio.

2 Véase Edey (1971).

${ }^{3}$ En algo más de una década han sido muy abundantes los trabajos de investigación histórica que se han elaborado. Destacan, junto a otros autores, $H$. Thomas Johnson y Alfred D. Chardler. En el citado libro de Johnson y Kaplan (1988) pueden encontrarse las referencias correspondientes. 


\section{Distintos tipos de información empresarial}

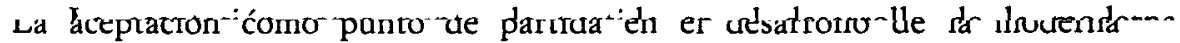
contabilidad de los dos procesos que acabamos de citar no significa que ambos sistemas de información contable, externa e interna, estén separados; tampoco que hayan seguido una evolución completamente independiente. Lo que sí pone de manifiesto este enfoque dual son las diferencias de la información contable, dependiendo de la finalidad con la que haya sido elaborada. La información va a responder a las distintas necesidades de las personas interesadas en aspectos diferentes de la entidad (por ejemplo, un accionista y un director operativo).

A nuestro juicio, el planteamiento central de Johnson y Kaplan, en el libro que da pie a esta nota, es el de la distinción entre diferentes informaciones empresariales que sirven para fines que son distintos. Dichos autores concluyen, en base a la información histórica reciente, que:

a) Es incierto que la contabilidad de gestión sea un fenómeno más reciente que la moderna contabilidad financiera.

b) Es incierto que la contabilidad de gestión surgiera para cubrir las demandas de información sobre los costes de los inventarios que debían figurar en los informes financieros externos.

Conclusión que haríamos mal en tildar de baladí, puesto que contrasta con lo que sigue siendo un enfoque aceptado, incluso en los años ochenta, sobre el surgimiento de la contabilidad de gestión. Una ojeada a los principales manuales recientes de esta disciplina nos mostraría dicho contraste.

Una vez admitido, pues, que la información contable empresarial puede responder a demandas diferentes, se plantea el problema de analizar en qué medida la respuesta ha sido en el pasado y es actualmente acorde a la información demandada. Para este análisis nos será útil explicitar la teoría sobre la empresa que estamos manejando. Teoría que habrá de explicar el papel de la empresa, y en función de este papel, su organización y funcionamiento nos plantearemos la cuestión de los «indicadores», esto es, las características que debe reunir la información contable.

En la teoría neoclásica que supone la existencia de información perfecta, la empresa prácticamente se limita «a resolver el problema matemático que determina la mezcla productiva óptima». Con esta perspectiva «más que una organización, la empresa es en realidad un mecanismo (...) que, al igual que el sistema económico de mercado en el que se halla inmerso se autorregula de forma automática» ${ }^{4}$. Por lo que este enfoque no puede resultar muy fruc-

\footnotetext{
‘ Suárez Suárez (1988), p. 15.
} 
tífero para el análisis de la información que necesita una organización empresarial.

Actualmente se puede afirmar que no existe una teoría sobre la empresa que sea generalmente aceptada. Las líneas de investigación son múltiples, destacando para gran parte de ellas el denominador común de compartir un enfoque de la entidad como estructura organizativa o marco donde se coordina o dirige a unos elementos (humanos, financieros, etc.) que están interrelacionados.

Herbert A. Simon ha destacado el papel pionero que tuvieron los escritores de la llamada tradición institucionalista (John R. Commons, Chester I. Barnard, etc.) que compartían la convicción de que la teoría sobre la empresa debía ser reformulada para que tomase en consideración las estructuras sociales y legales entre las que tienen lugar transacciones de mercado ${ }^{5}$. Entre los desarrollos de la teoría de la empresa que actualmente aportan investigaciones fecundas se sitúan los de la «economía de la información» $y$ el planteamiento de "la racionalidad limitada» ${ }^{6}$.

En este trabajo vamos a abordar la cuestión de la relevancia de la información contable, es decir, su capacidad para aportar los datos que le son demandados, utilizando los enfoques de dos teorías que analizan la organización empresarial en tanto que sistema en el que tiene un relieve especial el subsistema de procesamiento de la información y toma de decisiones. Se trata de la teoría de la agencia y de la teoría de los costes de transacción.

\section{La empresa como concierto}

La teoría de la agencia, desarrollada con el propósito de construir una teoría formal de la demanda de información para la empresa, al contemplar a ésta como algo diferente a una entidad monolítica, nos servirá para reflexionar tanto sobre las organizaciones como sobre la información que éstas producen.

Como es bien conocido, la teoría de la agencia analiza la empresa como una organización multipersonal. En este marco la empresa la constituyen un conjunto de contratos entre agentes económicos, cada uno de los cuales está motivado únicamente por su interés propio. Teoría que al reconocer los intereses económicos de los usuarios de la información contable señala explícitamente que los sistemas de información contable hacen algo más que generar

\footnotetext{
s Véanse Simon (1979), pp. 499-500, y Barnard (1938).

- Véase Simon (1979), pp. 500-501 y pp. 508-509; es de interés, asimismo, el artículo de Simon (1986).
} 
señales para los decisores. Esta teoría ha tenido diversos desarrollos ${ }^{7}$, y el que nos va a interesar ahora es el que en base a esta teoría analiza la información contable dirigida a terceros, o externa.

Aunque se hayan considerado en la empresa múltiples relaciones que entran dentro de la teoría de la agencia (como son las existentes entre propietarios y directivos, clientes y directivos, empleados con funciones directivas y sin ellas, alta dirección y dirección a otros niveles, etc.) vamos a considerar, en este artículo, que en el dominio de las grandes compañías dirigidas por profesionales, los protagonistas centrales y usuarios principales de la información son los gestores y los propietarios del capital. Entre ambos se ha descrito una vieja pugna, sobre la base de la posible indiferencia de los directivos hacia los objetivos de los propietarios; pugna que motivó terribles profecías, desde que se consolidó el tipo de empresa multidivisional, como fueron las de A. Berle y G. Means en 1932 con el libro The Modern Corporation and Private Property ${ }^{8}$, y que continuó a través de grandes popularizadores (como J. K. Galbraith en The New Industrial State ${ }^{9}$ ) hasta nuestros días, en que se podría entender que se aborda desde el contexto más amplio de la teoría de la agencia.

Junto con los aspectos de la legislación mercantil, a los que se hizo mención, el desarrollo del mercado de valores en Inglaterra creó una demanda de información contable para terceros o externa, que desde mediados del siglo XIX se concretará en informes financieros auditados por contables independientes. En aquel país los auditores habían establecido su presencia profesional con casi cincuenta años de ventaja sobre lo que tendría lugar en Estados Unidos ${ }^{10}$, cuando para explotar las grandes reservas americanas de capital externo a la empresa los directivos americanos comenzaron a presentar informes financieros auditados.

Hay que poner de relieve que, en la tradición anglosajona, la auditoría ha ido asumiendo diversos papeles, al ir más allá de la mera verificación de cuentas externa a la empresa y así ha establecido un conjunto de «principios generalmente aceptados» que van a subyacer en la información sobre la marcha de la empresa ( $y$ que son independientes, por ejemplo, de los criterios fiscales para el tratamiento de la contabilidad de la empresa). La auditoría viene a definir criterios homogéneos para el conjunto de las empresas, que sirven para la realización de informes financieros.

? Véanse los artículos de Carrasco Fenech (1987) y (1988).

Berle y Means (1932).

Galbraith (1967).

${ }^{10}$ No ocurrió, sin embargo, un liderazgo de este género con la contabilidad para la gestión interna, a pesar del desarrollo industrial, más temprano en el caso inglés. Véase una explicación sobre este punto en Johnson y Kaplan (1988), pp. 143-145. 
Cabría señalar aquí que la tradición española, en lo que respecta a las normas contables, se fundamenta en otra tradición, la continental europea, que confía el papel de uniformización contable a la propia Administración. Esta tradición está empezando a transformarse en el sentido de un acercamiento a la anglosajona, aunque en la práctica convivan ambas".

La información sobre la empresa, que van a aportar los directivos a los accionistas, en el enfoque anglosajón, se elabora de acuerdo con unos "principios generalmente accptados». Al utilizar dichos principios, la información financiera externa habrá de sujetarse a unos mínimos de calidad y cantidad preestablecidos. $Y$ en este sentido la teoría de la agencia ${ }^{12}$ resalta que los principios aceptados tienen una función de compromiso entre directivos y accionistas, ya que permite, siendo los propios directivos los que presentan la información sobre su gestión, un control por parte de los accionistas de lo que ha supuesto la actividad de los directivos. Por lo que la existencia de unas normas de general aceptación actuaría como freno a un hipotético comportamiento de los directivos que fuera inconsistente con los intereses de los propietarios.

La intervención de unos auditores independientes encargados de la revisión contable actuará como un control adicional, observando el cumplimiento de las normas contables comunes. La consecuencia final estaría en la confianza que aporta al mercado de capitales la información financiera elaborada de esta forma por las compañías, lo que vendría a facilitar la asignación de recursos a través de este mercado.

"Frente a los arquetipos mencionados como «tradición anglosajona» y «tradición continental», la situación concreta española se podría plantear brevemente así (por otra parte, en el núm. 31 de la revista Economistas, Colegio de Madrid, abril-mayo 1988, puede verse esta situación descrita con detalle): Es sabido que, junto con las ya existentes normas contables sobre sociedades mercantiles y normas fiscales, en 1973 el Ministerio de Hacienda publicó un Plan General Contable, siguiendo de cerca la experiencia francesa. Plan que sigue vigente en nuestros días. Sin cmbargo, la aprobación en 1978 de la Ley de Impuesto de Sociedades y, sobre todo, del Reglamento correspondiente en 1982 hace aparecer lo que bien se podría considerar como un plan contable, que, aunque con finalidad fiscal, ha tenido una repercusión práctica mucho más ampiia. Paralelamente, la internacionalización de la economía española hace progresar el enfoque de una «autorregulación contable», realizada por cuerpos de profesionales independientes, siguiendo los ejemplos anglosajones; con el resultado de la creación de la AECA (Asociación Española de Contabilidad y Ad. ministración de Empresas), que comienza a contribuir al consenso sobre normas contables. Este panorama se completa con el surgimiento recicnte, y en algunos casos todavía en proceso, de una nueva legislación -adaptación, en algunos casos, a directivas comunitarias europeas (la IV, VII y VIII) - sobre auditoría, sociedades mercantiles, mercado de valores, fondos de pensiones, grupos de sociedades, etc. Cambios legales que afectan a la información empresarial. El Ministerio de Economía, a través del Instituto de Planificación Contable, ha elahorado un proyecto de nuevo plan general de contabilidad, acorde con dichos cambios legales.

12 Véase el artículo de Gordon y Hamer (1983). 


\section{Compañias y mercados}

Paralelamente a la información para uso externo surge la necesidad de disponer de datos que permitan controlar los procesos internos y tomar decisiones que afectan a:

a) La planificación estratégica (sobre objetivos de la empresa y recursos a utilizar para alcanzar los objetivos).

b) El control de gestión (sobre la obtención y utilización eficiente y eficaz de los recursos).

c) El control de operaciones (sobre el desarrollo de tareas específicas de compras, producción, etc.).

El conjunto de estas operaciones tiene una orientación interna, y diferente, por tanto, de la orientación de la contabilidad para uso externo, que se ha tratado en el apartado anterior. En el caso de una contabilidad para la gestión, no se espera que se adhieran todas las empresas a unas normas comunes, y no hay principio que les sea impuesto, al tratarse de una información sobre aspectos relacionados con la calidad de los productos, los niveles de inventario, el rendimiento de los factores empleados, los costes de los procesos y de los productos, el proceso de $\mathrm{I}+\mathrm{D}$, los resultados calculados bajo los enfoques pertinentes según el caso, la política de precios de venta, los precios de transferencia interna, la gestión presupuestaria, los indicadores de beneficio en función de las inversiones, y un largo etcétera. Todo ello es la información sobre la empresa que abarca la contabilidad de gestión como disciplina académica ${ }^{13}$.

Las características de la propia empresa establecerán la demanda de información para la gestión interna. Así ocurrió históricamente desde. el surgimiento de la moderna contabilidad de gestión, cuando a través de los procesos de integración entre algunas empresas, en lugar de confiar a mercados externos la dirección del intercambio económico, comenzaron a encadenar procesos antes separados y a controlarlos de forma interna.

Los empresarios de los nuevos procesos se plantearon las ventajas potenciales de dirigirlos de forma jerarquizada en la propia compañía en lugar de utilizar el mercado para intercambiar los productos resultantes de los distintos segmentos del proceso. En EE. UU. este proceso se inicia en el siglo xix en

${ }^{13}$ En la amplia nómina de autores que han trabajado en este campo habría que citar nombres como Shillinglaw, Horngren, Dearden y otros muchos. A dicha lista se añade, en esta década, Robert $S$. Kaplan, quien, junto a sus artículos y al libro que publicó con Johnson (1988), ha publicado una aportación teórica con el libro Advanced Management Accounting (1982). 
las fábricas textiles mecanizadas de algodón que aparecieron después de 1812, las cuales seguían empleando la información de los precios de mercado en sus transacciones externas con consumidores y proveedores, a la vez que crearon el primer ejemplo de la contabilidad de gestión con un sistema para medir costes de explotación centrado en el rendimiento del trabajo y de las materias primas, destinado a racionalizar los procesos coordinados internamente. Se ha señalado que

«la contabilidad de gestión centró la atención de la gente en las ganancias potenciales derivadas de la coordinación interna del intercambio económico, alentando a los directivos empresarios a aumentar el tamaño de las empresas" ${ }^{14}$.

La creación de las grandes compañías ferroviarias, en EE. UU. a partir de 1840, marcó el comienzo de las grandes empresas en aquel país. Entre las muchas soluciones empresariales originales que aportaron, figuran unos sistemas contables internos acordes con la gran escala de las entidades que había que controlar. Y con la aparición a fines del siglo XIx de las empresas siderúrgicas del acero, la demanda de datos para la gestión interna se centra en sistemas de contabilidad de costes de producción directos y margen de contribución con los que plantearse precios que aseguraran una demanda suficiente para mantener la planta funcionando a plena capacidad.

Un último tipo de empresa unisectorial surge en el último cuarto del siglo pasado en la economía americana: el distribuidor a gran escala, que viene asociado al desarrollo de bienes producidos en masa y de forma estandarizada, y al desarrollo de los ferrocarriles y el telégrafo. Estos distribuidores masivos desarrollaron sistemas de contabilidad de gestión nuevos para el control de una estructura que comenzaba a descentralizarse (margen bruto, tasas de rotación del inventario, etc.).

Las prácticas contables de gestión de las nuevas empresas industriales que acabamos de señalar buscaban - a través de distintos indicadores- formas de evaluar la eficiencia con la que estaban utilizando los recursos en sus procesos dirigidos internamente (procesos que, de otra forma, hubieran sido coordinados a través del mercado o no se hubieran producido) ${ }^{15}$.

${ }_{14}$ Johnson y Kaplan (1988), p. 36.

is Si hubiera que comparar este proceso, que tuvo lugar en EE. UU., con el proceso que se produjo en Inglaterra encontraríamos diferencias notables. A pesar del papel destacado inglés en el desarrollo industrial, su contabilidad de gestión se desarrolló posteriormente $y$ en menor medida que en EE. UU. La investigación actual señala la mayor efectividad de los mercados ingleses para coordinar distintos procesos de producción. Véanse las referencias bibliográficas a este respecto en Johnson y Kaplan (1988), cap. V, no. tas 43 y 48 . 
Con el surgimiento a principios del siglo $\mathrm{xx}$ de las empresas multisectoriales.americanas, fusionando dos o más actividades separadas, se puso de manifiesto que las ganancias potenciales se producirían sólo si surgía un tipo de organización de las empresas que permitiera coordinar actividades hacia objetivos comunes. Como se ha puesto de relieve:

«El complejo sistema de intercambios internos, característicos de la empresa multisectorial mitiga el efecto de la incertidumbre del mercado. Irónicamente, esta complejidad crea otra fuente de incertidumbre. Como la información sobre sus complejos procesos internos es difícil de asimilar, una empresa integrada puede ahogarse en un mar de ineficiencia burocrática, perdiendo sus beneficios potenciales» ${ }^{16}$.

El análisis histórico de las compañías multisectoriales americanas muestra el desarrollo de dos tipos de estructuras organizativas:

a) Inicialmente, los sistemas centralizados. Estos dividen las operaciones de la empresa en departamentos separados, con sus respectivos directores llevando su actividad según criterios de eficacia y de eficiencia, con el mismo tipo de técnicas que emplea una empresa unisectorial (costes, rendimientos; economías de escala). La oficina central coordina como si se tratara de una unidad a los departamentos hacia metas comunes, integrando todas las actividades, para llegar de este modo a unos resultados mayores de los que se podrían obtener en el mercado con cada departamento operando de forma independiente.

b) Posteriormente, en la segunda década del siglo $\mathrm{xx}$ en EE. UU., surgen las empresas multidivisionales, señaladas por algún autor como la innovación más importante del capitalismo americano en el siglo xx. Este tipo de organización empresarial, que llega hasta nuestros días con los conglomerados multidivisionales, fue creada para hacer frente a la pérdida de control directivo sobre la empresa derivada de unas compañías multisectoriales cada vez más complejas.

Las Organizaciones multidivisionales descentralizadas tienen el mérito de preservar la vitalidad de la gran empresa, al gestionar la diversidad de actividades de una forma menos costosa, descentralizando al máximo la actividad de cada división que se convierte en algo próximo a una compañía independiente (a excepción de algunos aspectos financieros); pero que, sin em-

\footnotetext{
16 Johnson y Kaplan (1988), p. 72.
} 
bargo, sigue unas metas de beneficio comunes para el conjunto de la corporación.

Tanto en las empresas multisectoriales centralizadas como en las multidivisionales, la gestión del capital invertido se convirtió en una de las fuerzas matrices de la empresa. El indicador ROI, «retorno de la inversión» (que compara los resultados con la inversión necesaria), facilitó la coordinación de actividades de la compañía. En las empresas integradas verticalmente no bastaba con conocer la eficiencia de los procesos una vez el capital se había invertido, había que asignar capital a las actividades.

Se ha señalado que si los primeros sistemas contables de gestión crearon indicadores que les permitieron descubrir oportunidades de ahorro de trabajo, la empresa integrada verticalmente del siglo $\mathrm{xx}$, a través de la medida del retorno de la inversión, buscaba las oportunidades productivas para invertir el capital en la propia compañía considerada globalmente ${ }^{17}$. Es decir, aplicaba el principio de que

«no había gastos para ampliaciones de equipo si la misma cantidad de dinero podía aplicarse a un propósito mejor en otra rama del negocio de la empresa» ${ }^{18}$.

Tanto en el caso de la empresa multisectorial centralizada (en la cual la alta dirección asignaba las nuevas inversiones) como en el caso de la empresa multisectorial divisionalizada (en la que los directores de división tenían delegada la responsabilidad de usar capital de forma eficiente, dentro de unos parámetros), el uso imaginativo de la información sobre el rendimiento del capital invertido hizo posible que la dirección «suplantara a los mercados de capital en la decisión de asignación de recursos" ${ }^{19}$.

La tecnología de tratamiento de datos disponible en los primeros treinta años del siglo $\mathrm{xx}$ ha sido considerada como un factor determinante de la elección del indicador «retorno de la inversión», en lugar de emplear otros indicadores disponibles en la teoría. Con este indicador escogido se sintetizaban señales dispersas y se relacionaban con el rendimiento global de la empresa, y todo ello sin encarecer el coste de coordinar las diferentes actividades internas, lo que hubiera podido limitar la dimensión de las empresas.

El enfoque sobre el desarrollo de la contabilidad de gestión, adoptado por Johnson y Kaplan, y que hemos seguido hasta aquí en este apartado y en particular la información que la contabilidad de gestión proporciona sobre las oportunidades de ganancia que se ofrecen al operar en la estructura jerár-

\footnotetext{
17 Véase Johnson y Kaplan (1988), p. 95.

16 Johnson y Kaplan (1988), p. 77.

19 Johnson y Kaplan (1988), p. 90.
} 
quica de la empresa en lugar de operar en el mercado de productos o de capitales, está inspirado, como es manifiesto, en la aportación ya clásica sobre la empresa de Ronald $\mathrm{H}$. Coase ${ }^{20}$, y que ha sido desarrollada posteriormente por otros autores, entre ellos Oliver Williamson ${ }^{21}$, y no deja de ser una buena ilustración de este planteamiento sobre la base del análisis histórico. Recordemos, a este respecto, la afirmación de Coase de que

«fuera de la empresa las variaciones de precios dirigen la producción, la cual se coordina a través de un conjunto de intercambios en el mercado. En el interior de la empresa estas transacciones de mercado quedan eliminadas y el lugar de los intercambios con la complicada estructura de mercado lo sustituye el empresariocoordinador quien dirige la producción. Es manifiesto que se trata de métodos alternativos para coordinar la producción»" ${ }^{22}$.

El límite a la dimensión de las actividades empresariales los sitúa Coase en los llamados «rendimientos decrecientes del management»" ${ }^{23}$, y hace que la validez de uno u otro método de asignación sea relativa y pueda estar sometida, por tanto, a revisión, con la consiguiente oscilación de un método a otro.

\section{Relevancia de la información empresarial}

También ha sido planteado por el libro de Johnson y Kaplan, que ha servido de estímulo a este artículo, y ésta es quizá la tesis más escandalosa de aquella obra, que

«los sistema de control de gestión y contabilidad de costes contemporáneos $(\ldots)$ ya no dan señales ajustadas sobre la eficiencia y la rentabilidad de las transacciones gestionadas internamentem.

$\mathrm{Y}$, como consecuencia,

${ }^{20}$ Coase (1937). Es de interés, asimismo, el artículo de Edwards (1937). Sobre el planteamiento más amplio de los costes de oportunidad, véase Coase (1938).

${ }_{21}$ Véanse las referencias bibliográficas sobre $O$. Williamson, y sobre las evidencias em. píricas obtenidas con investigaciones de otros autores, en Johnson y Kaplan (1988), pp. 122-125. La obra histórica de síntesis que impulsó en buena medida la investigación posterior es la de Chandler (1987).

${ }^{22}$ Coase (1937), p. 388.

${ }^{23}$ Coase (1937), p. 388. 
«sin recibir información apropiada sobre costes y rentabilidad, la habilidad de la "mano visible" para gestionar de forma efectiva la riada de transaciones que ocurren en una jerarquía compleja se ve seriamente comprometida» ${ }^{24}$.

La preocupación que late detrás de estas palabras surgió inicialmente al comprobar a comienzos de los años cchenta la agudización de la pérdida de competitividad de algunos procesos productivos industriales de las empresas americanas, bajo la competencia de empresas japonesas o europeas. Un conjunto de autores puso, además, de manifiesto que los indicadores diseñados para la gestión empresarial no daban en algunos casos una idea apropiada de la situación real ${ }^{25}$; en especial, se planteaba que no informaban adecuadamente cuando la competencia en lugar de asentarse sobre una producción barata y masiva de productos estandarizados se fundaba sobre aspectos nuevos, como, por ejemplo, niveles de calidad total en los productos (zero-defect systems); alta rotación de inventarios (con sistemas just-in-lime); sistemas de producción flexible con pequeñas series «a medida»; innovación en los procesos productivos y en los productos; especialización en productos de alta tecnología con ciclos de vida cortos, etc.

Además, se ponía de manifiesto que, a pesar del desarrollo de un buen nivel académico para la contabilidad de gestión en las décadas anteriores, los sistemas de información realmente implantados en las empresas no proporcionaban los datos precisos para poder responder a las situaciones nuevas en los mercados actuales, ni a las nuevas tecnologías, ni a los nuevos sistemas de organización.

La información que quiera ser relevante a partir de los años ochenta - Johnson y Kaplan nos proponen- deberá adaptarse al nuevo entorno por medio de la integración en la información de señales sobre los procesos cada veZ más decisivos de $\mathrm{I}+\mathrm{D}$; deberá plantearse el análısis de la rentabilidad a larog plazo más que a corto plazo, con atención al ciclo vital de los procesos productivos y de los productos. Se señala también la importancia de los indicadores no financieros en el análisis de la actividad de la empresa, y un sistema de incentivos y participación acorde con la nueva información. Las nuevas tecnologías afectan a la capacidad de procesar información, lo que supone una necesidad de explorar nuevas posibilidades y nuevos enfoques (por ejemplo, desagregando información; empleando distintos datos de coste o de resultados para análisis diferentes; con la posibilidad de utilizar los criterios de valoración más apropiados en cada caso, etc.).

24 Johnson y Kaplan (1988), p. 200.

25. Es significativo el artículo de Kaplan (1983). 
El enfoque reseñado urge, por tanto, a todo un programa de adaptación de la contabilidad de gestión a nuevas realidades, lo que supone también un proceso de investigación ${ }^{26}$, si se quiere recuperar la «relevancia perdida». Significativamente el título original del libro de Johnson y Kaplan, que suscitó estas líneas, es Relevance Lost. The Rise and Fall of Management Accounting; y sus autores, tanto en el título como en la cita de Milton (tomada de Paradise Lost) que abre el libro, han expresado irónicamente un símil con la «caída» y expulsión del Paraíso. Quizá, de este libro, la parte que necesitará mayor atención en el futuro, por ser la menos profunda desde nuestro punto de vista, sea la de la "caída» y el análisis detallado de sus causas, sobre lo que resta por hacer un trabajo profundo de investigación histórica. En cualquier caso queda pendiente una importante tarea si, por seguir refiriéndose a Milton, se tiene la vista puesta en el Paradise Regained.

Por otra parte, no es únicamente la contabilidad de gestión la que se ve sometida a nuevas demandas pujantes; la contabilidad externa para terceros experimenta, aunque con una intensidad inferior, el cambio económico del entorno (internacionalización del comercio, conglomerados de empresas, cambio en los mercados financieros, armonización internacional de la información, desregulación de mercado, etc.); procesos que supondrán también adaptación al nuevo entorno.

Estos procesos recientes de cambio económico hacen que surja ante nosotros lo que parece ser una buena oportunidad para contrastar - en otro orden de cosas - las teorías de la empresa - y, por tanto, sobre su dimensión, organización, objetivos, funcionamiento- que hemos venido utilizando para explicar las demandas externas o internas de información contable.

${ }^{26}$ Entre las múltiples aportaciones recientes pueden consultarse: Williams y otros (1987), Jonsson (1987), Brown y Sprohge (1987), Mevellec (1988), Escudero (1987), Kumpe y Bolwijn (1988) y Kaplan (1988). 


\section{BIBLIOGRAFIA}

Barnard, Ch. I. (1938): The Functions of the Executive, Cambridge, Mass. (hay traducción castellana: Las funciones de los elementos dirigentes, Madrid, Instituto de Estudios Políticos, 1959).

Berle, A., y Means, G. (1932): The Modern Corporation and Private Property, Nueva York, Macmillan.

BRoWN, R., y SPROHGE, H.D. (1987): “Governmental Managerial Accounting: What and Where is It?", Public Budgeting and Finance, otono.

Buchanan, J. M., y Thirl.by, G. F. (1973): L. S. E. Essays on Cost, Nueva York, New York University Press.

Carrasco Fenech, Francisco (1987): "Teoría de la agencia y contabilidad: una primera aproximaciónm, Cuadernos de Investigación Contable, Universidad de Sevilla, otoño.

- (1988): "La contabilidad por áreas de responsabilidad desde una perspectiva de agenciam, Cuadernos de Investigación Contable, Universidad de Sevilla, primavera.

CoAse, R. H. (1937): «The Nature of the Firm", Economica, noviembre.

- (1938): «Business Organization and the accountant», editado en Buchanan, J. M., y ThirlBy, G. F. (1973): L. S. E. Essays on Cost, Nueva York, New York University Press.

ChANDLER, Alfred D. (1987): La Mano visible. La revolución en la dirección de la empresa norteamericana, Madrid, Centro de Publicaciones del Ministerio de Trabajo y Seguridad Social.

EDEY, Harold (1971): "The True and Fair View», Accountancy, agosto.

EDWARDS, R. S. (1937): "The Rationale of Cost Accounting», editado en Buchanan, J. M., y Thirlby, G. F. (1973): L. S. E. Essays on Cost, Nueva York, New York University Press.

Escudero, L. F. (1987): «Planificación de la producción en fabricación flexible», Boletin de Estudios Económicos, Deusto, agosto.

Galbraith, J. K. (1967): The New Industrial State, Boston, Houghton Miffin.

Gordon, L. A., y Hamer, M. (1983): "GASB's Survival Potential: An Agency Theory Perspective», Public Budgeting and Finance, primavera.

Johnson, H. T., y Kaplan, R. S. (1988): La contabilidad de costes. Auge y caida de la contabilidad de gestión, Barcelona, Plaza y Janés (v. o.: Relevance Lost. The Rise and Fall of Management Accounting, Boston, Harvard Business School Press, 1987).

Jonsson, Sten (1987): «Nuevas tecnologías y contabilidad de gestión», Cuadernos de Investigación Contable, Universidad de Sevilla, otoño.

Kaplan, Robert S. (1982): Advanced Management Accounting, Englewood Cliffs (Nueva Jersey), Prentice-Hall.

- (1983): «Measuring Manufacturing Performance: A New Challange for Managerial Accounting Research», Accounting Review, octubre.

- (1988): «One Cost System Isn't Enough», Harvard Business Review, enero-febrero.

Kumpe, T., y Bolwijn, P. T. (1988): «Manufacturing: The New Case for Vertical Integration», Harvard Business Review, marzo-abril.

Mevellec, Picrre (1988): «La comptabilité analytique face à l'évolution technologique», Revue Française de Gestion, núm. 67.

Srmon, H. A. (1979): «Rational Decision Making in Business Organizations», American Economic Review, septiembre.

- (1986): «Rationality in Psychology and Economics», Journal of Business, vol. 59, núm. 4.

SuÁrez Suńrez, A. S. (1988): La teoría de la empresa y el estado del bienestar, Madrid, Facultad de CC. EE. y EE. de la UCM. Texto fotocopiado de la conferencia pronunciada.

Williams, K., y otros (1987): «The End of Mass Production», Economy and Society, agosto. 